\title{
Multiple metastases in a novel LNCaP model of human prostate cancer
}

\author{
MINHONG ZOU*, JU JIAO*, QIONG ZOU, YAN XU, MUHUA CHENG, JIEHUA XU and YONG ZHANG \\ Department of Nuclear Medicine, The Third Affiliated Hospital, Sun Yat-sen University, \\ Guangzhou, Guangdong 510630, P.R. China
}

Received December 7, 2012; Accepted January 30, 2013

DOI: $10.3892 /$ or.2013.2305

\begin{abstract}
Metastasis is a frequent and lethal consequence of prostate cancer. Current treatments for metastasis are palliative only. Thus, experimental animal models of metastatic prostate cancer are required for investigations of its pathogenesis and for the development of treatment strategies; however, few models exist at present. In the present study, the LNCaP prostate cancer cell line was co-transfected with a PGK-luciferase-GFP lentivirual vector (LNCaP-luc). Repeated subcutaneous injections of LNCaP-luc cells with Matrigel in nude mice followed by isolation of the cells from tumors resulted in the generation of the LNCaP1-luc cell line. We used CCK-8 and Transwell migration assays, western blot analysis and polymerase chain reaction to detect differences in the characteristics between the LNCaP-luc and LNCaP1-luc cells, and used LNCaP cells to generate a mouse model of metastatic prostate cancer by intracardiac injection. Metastasis was evaluated by bioluminescence imaging, and histological and immunohistochemical staining. The characteristics of the LNCaP1-luc cells differed from those of LNCaP cells, and LNCaP1-luc cells showed increased cell proliferation, cell invasion, tumorigenicity and metastasis potential, and underwent epithelial-mesenchymal transition. In addition, the LNCaP1-luc cells induced multiple metastases in mice when injected into the left cardiac muscle.
\end{abstract}

\section{Introduction}

Prostate cancer is the most common cancer in males in Europe and in the United States, and the third leading cause of death from cancer in Europe (1), resulting in 903,500 new cases and 258,400 deaths annually; $80 \%$ of these men succumb to bone metastatic cancer (2). The multistep process of carcinogenesis of prostate epithelial transformation is the transition

Correspondence to: Professor Yong Zhang, Department of Nuclear Medicine, The Third Affiliated Hospital, Sun Yat-sen University, 600 Tianhe Road, Guangzhou, Guangdong 510630, P.R. China

E-mail: zy5040@163.com

*Contributed equally

Key words: LNCaP cells, epithelial-mesenchymal transition, animal model, multiple metastases from an androgen-dependent non-metastatic phenotype to a more malignant metastatic androgen-independent phenotype (3). As a consequence, nearly 30,000 individuals die of bone metastatic cancer every year in the US alone (4). These figures demonstrate the tragic contribution of bone metastatic cancer to morbidity and mortality. Thus, novel therapies for the prevention and treatment of bone metastatic cancers are imperative. Due to the protracted natural progression of prostate cancer, clinical and epidemiological studies alone may not provide the knowledge necessary to design strategies for preventing, predicting and treating metastatic disease (5).

Therefore, to gain further insight into human prostate cancer progression and metastasis, well-defined in vivo models that mimic the different aspects of the natural course of disease progression are essential. However, the field of prostate cancer research continues to be hindered by the lack of relevant preclinical models with which to study tumorigenesis and develop effective prevention and therapeutic interventions (6). The most effective models are those that can be used to mimic changes in human disease, that can be utilized to ask questions that explain observed phenomena, and that are predictive of therapeutic efficacy (7). Several mouse models have been developed; these require injection or implantation of cancer cells into various locations, such as subcutaneous tissue, intra-osseous injection or injection into the left ventricle of the heart. Additionally, carcinomas can be induced by either chemical or physical agents (4,6,8-10). Although valuable for studying the growth, physiology and metastasis of metastatic processes in prostate cancer, these models are inadequate to provide a full understanding of the disease (11).

$\mathrm{X}$-ray examination is the technique most commonly used to detect bone metastases. Other techniques are also used to characterize bone metastases in animal models, such as microcomputed tomography, positron-emission tomography and scintigraphy. However, cancer tends to be diagnosed at a later stage, reducing access to suitable therapeutic facilities and drugs (12). Therefore, more sensitive methods are needed to detect cancer at the very early stages. Bioluminescence imaging is an emerging method that allows visualization of cancer of any stage, including growth of the primary tumor, tumor cell motility and invasion, and evaluation of the cytotoxicity of therapeutics. Bioluminescence imaging leads to increased detection sensitivity and more accurate quantification of metastases $(13,14)$. 
Since the model we describe here closely mimics the natural history of human prostate cancer, it is useful for future studies of the mechanisms and therapy of prostate tumor progression and metastasis. In addition, animal models of metastasis have been used in drug development $(5,15)$.

\section{Materials and methods}

Tumor cell line. The LNCaP cell line was purchased from the Institute of Biochemistry and Cell Biology, Shanghai Institutes for Biological Sciences, Chinese Academy of Sciences, Shanghai, China. Cells were routinely cultured in RPMI-1640 medium (Gibco, Grand Island, NY, USA) supplemented with 20\% F12K (Irvine Scientific, Santa Ana, CA, USA), $100 \mu \mathrm{g} / \mathrm{ml}$ streptomycin, $100 \mathrm{U} / 1$ penicillin $\mathrm{G}$ and $15 \%$ fetal bovine serum (FBS) (Gibco) in a humidified atmosphere of $5 \% \mathrm{CO}_{2}$ at $37^{\circ} \mathrm{C}$. The cells were passaged weekly at a 1:3 dilution using $0.05 \%$ trypsin (Gibco).

Cell transfection. LNCaP cells were co-transfected with the PGK-luciferase-green fluorescent protein (GFP) lentiviral vector containing the PGK expression vector plasmid encoding luciferase and GFP (Invitrogen, Carlsbad, CA, USA), using the LNCaP-luc cationic liposome method. GFP expression was observed by fluorescence microscopy after $48 \mathrm{~h}$, and transfection efficiency was monitored by flow cytometry.

Subcutaneous injection into nude mice. The LNCaP-luc cell line was fed with fresh culture medium $48 \mathrm{~h}$ before cell inoculation Cells were harvested and then incubated in culture medium for $3 \mathrm{~min}$ and suspended in phosphate-buffered saline (PBS) immediately before inoculation. Cells $\left(2 \times 10^{6}\right)$ in $0.1 \mathrm{ml}$ PBS mixed with $0.1 \mathrm{ml}$ Matrigel were injected subcutaneously (s.c.) into the groin of nude mice. Tumor growth was monitored by external caliper measurements [tumor size $=$ (length $\mathrm{x}$ width $\mathrm{x}$ height) $\mathrm{x} 0.52]$.

Statement of ethics. Male BALB/c nude mice were purchased from Beijing Weitong Lihua (Beijing, China). Experiments were carried out according to the National Institutes of Health Guide for the Care and Use of Laboratory Animals and were approved by the Bioethics Committee of Sun Yat-sen University. Mice were maintained in laminar flow boxes under sterile conditions. Sterile water and food were provided. Mice were 4-5 weeks old when used for intracardiac injections of tumor cells and 5-6 weeks old when injected with tumor cells.

Generation of the LNCaPl-luc cell line. Animals were sacrificed when subcutaneous tumors grew to nearly $1 \mathrm{~cm}$ in diameter. Tumor tissues were harvested from the subcutaneous tissue under aseptic conditions. Briefly, tumors were minced into $1-\mathrm{mm}^{3}$ cubes, placed on small culture dishes, and immersed in tissue-culture medium. Tumor cells were outgrown together with host fibroblasts within 1.5 weeks. LNCaP-luc tumor cells, which adhered loosely to the plastic dishes, were enriched by washing the culture dishes with PBS. The pure LNCaP cell line (LNCaP1-luc) was obtained after 5-10 of these subculturing steps.
CCK-8 assay. LNCaP-luc and LNCaP1-luc cells were digested and dispensed into 96 -well plates at a density of $3 \times 10^{3} /$ well (100 $\mu \mathrm{l} /$ well) for the cell viability assay. After a 42-h incubation, $10 \mu \mathrm{l}$ of CCK-8 was added to each well for an additional $4 \mathrm{~h}$ at $37^{\circ} \mathrm{C}$. Plates were then shaken for $10 \mathrm{~min}$, and the optical density values at $450 \mathrm{~nm}$ were measured according to the manufacturer's instructions. All experiments were carried out in triplicate to ascertain reproducibility. Growth curves were generated from these data. The control group contained only cell-culture medium.

Transwell migration assay. Transwell migration assays were carried out using 24-well cell-culture plate inserts and Transwell filters with $8-\mu \mathrm{m}$ pores. Cells were cultured for $24 \mathrm{~h}$ in serum-free RPMI-1640 medium and then seeded $\left(1.5 \times 10^{5}\right)$ into the inner chamber with $300 \mu \mathrm{l}$ serum-free RPMI-1640 and $500 \mu \mathrm{l}$ of RPMI-1640 containing $10 \% \mathrm{FBS}$ in the outer chamber. Cells were allowed to migrate for $24 \mathrm{~h}$ at $37^{\circ} \mathrm{C}$ in $5 \% \mathrm{CO}_{2}$. The cells were stained with hematoxylin and washed twice in PBS. Non-migratory cells on the upper surface of the inner chamber were removed using cotton swabs. Cells in five random high-power microscopic fields (x100) per filter were counted.

Western blot analysis. Total protein of the LNCaP-luc and LNCaP1-luc cells was extracted using a cell lysis buffer containing $20 \mathrm{mM}$ Tris- $\mathrm{HCl}(\mathrm{pH} 7.6), 1 \%$ NP-40, $3 \mathrm{mM}$ EGTA, $3 \mathrm{mM}$ EDTA, $0.15 \mathrm{mM} \mathrm{NaCl}, 1 \mathrm{mM}$ phenylmethylsulfonyl fluoride, $5 \mathrm{mg} / \mathrm{ml}$ leupeptin and $20 \mathrm{mg} / \mathrm{ml}$ aprotinin. Homogenates were centrifuged at $12,000 \mathrm{rpm}$ for $20 \mathrm{~min}$ at $4^{\circ} \mathrm{C}$ and the supernatants were retained. The protein concentration was determined using a Bio-Rad DC Protein Assay (Bio-Rad Laboratories, Hercules, CA, USA). Equal amounts of the protein $(20 \mu \mathrm{g})$ were electrophoresed on $10 \%$ sodium dodecyl sulfate polyacrylamide gels and transferred electrophoretically to nitrocellulose membranes (Bio-Rad Laboratories). Following blocking with $5 \%$ non-fat milk in wash buffer at room temperature for $2 \mathrm{~h}$, the membranes were incubated with the primary vimentin, E-cadherin and GAPDH antibodies (1:1,000; Santa Cruz Biotechnology, Santa Cruz, CA, USA) at $4^{\circ} \mathrm{C}$ overnight. The membranes were then incubated for $1 \mathrm{~h}$ at room temperature with a horseradish peroxidase-conjugated secondary antibody at a 1:5,000 dilution. The membranes were investigated using Western Blotting Plus Chemiluminescence Reagent (Thermo Fisher Scientific, Rockland, IL, USA) and fluorography using X-ray film (Fuji, Tokyo, Japan).

Real-time reverse transcription-polymerase chain reaction (PCR) analysis. Total RNA was extracted from LNCaP-luc and LNCaP1-luc cells with the E.Z.N.A. ${ }^{\circledR}$ HPTotal RNA kit (Omega Bio-Tek, Norcross, GA, USA). cDNA was synthesized using the PrimeScript ${ }^{\circledR}$ RT reagent kit (Takara Bio, Shiga, Japan). After mixing the resulting complementary DNA template with vimentin, E-cadherin, Snail, Slug, or GAPDH primers, respectively, and Takara SYBR ${ }^{\circledR}$ Premix Ex Taq ${ }^{\mathrm{TM}}$, the quantitative real-time PCR reaction was performed on a LightCycler 480 Real-Time PCR system (Roche). Quantified expression values for individual genes were normalized to that of GAPDH. Changes in mRNA expression were expressed as fold-changes relative to the control. The gene-specific primers used in this 

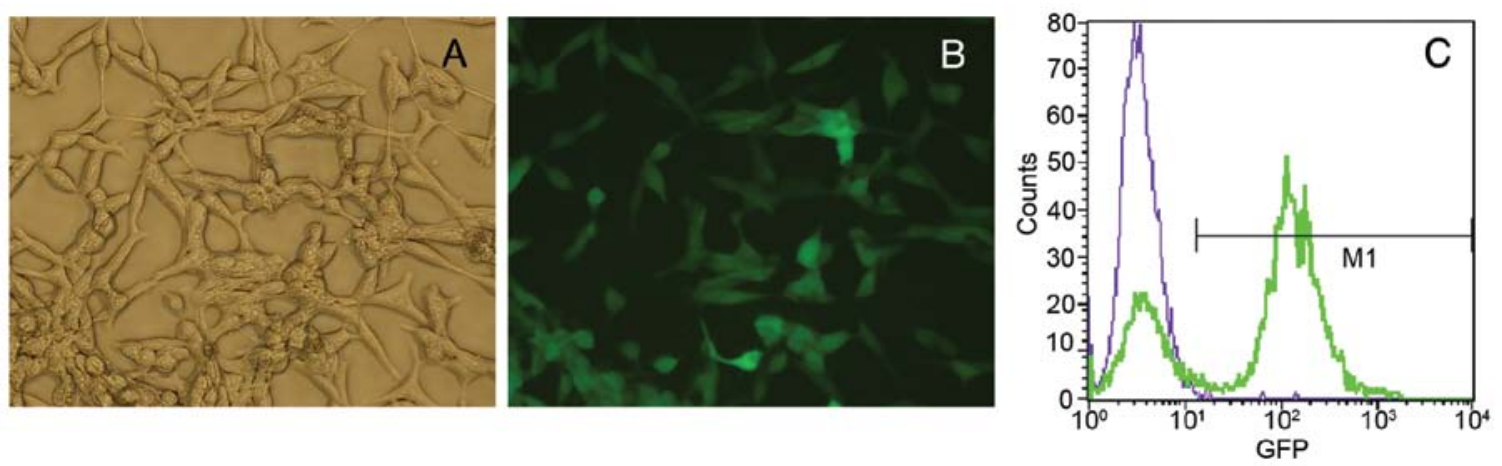

Figure 1. Transfection efficiency in the LNCaP cell line. Parental LNCaP cells were transduced with a recombinant lentiviral vector expressing luciferase and green fluorescent protein (GFP). (A and B) Fluorescence microscopy showed high GFP expression $24 \mathrm{~h}$ after lentiviral transduction. LNCaP-luc cells were polygonal in shape, and their fluorescence intensity was unequal. Compared to the parental LNCaP cells, the fluorescence profile of the LNCaP-luc cells showed that the entire population expressed GFP. (C) Expansion in culture after viral transduction was quantified by flow cytometry. The transfection efficiency of the cells approached $80 \%$, indicating complete transduction of the GFP-marker gene.

study were as follows. Primers for Vimentin, E-cadherin, Snail and Slug were as previously published (16). GAPDH sense was 5'-CACCCAGAAGACTGTGGATGG-3' and GAPDH antisense was 5'-GTCTACATGGCAACTGTGAGG-3'.

Intracardiac injection. Nude mice were anesthetized with ketamine, and LNCaP1-luc cells $\left(0.5 \times 10^{6}\right)$ suspended in $0.1 \mathrm{ml}$ PBS were injected into the left cardiac ventricle following a modification of the technique first described by Arguello et al (11). Successful injection was indicated retrospectively by pulsatile bright red blood in the $29 \mathrm{G}$ needle fitted with a 1.0-ml insulin syringe. Successful injection was also confirmed by the lack of abnormal tumor mass growth in the pleural cavity 3-4 weeks after injection.

Bioluminescence imaging. Mice were imaged for bioluminescence weekly for 4-6 weeks after intracardiac injection using the IVIS ${ }^{\circledR}$ Imaging system from Caliper (Alameda, CA, USA). Images were acquired and processed using the Living Image ${ }^{\circledR}$ version 4.2 software. Anesthesia was induced with inhaled isoflurane and maintained with $2 \%$ isoflurane mixed with oxygen/nitrogen via a nose cone. D-luciferin $(3 \mathrm{mg}$ dissolved in water) was administered at $150 \mathrm{mg} / \mathrm{kg}$ in DPBS via intraperitoneal injection. Optical imaging was carried out 10-15 min later. One to three mice were imaged at a time, and the tails, feet and paws were taped to prevent movement artifacts. One-minute acquisitions were conducted at the highsensitivity setting of the instrument software. Background was subtracted using an automated feature of the program.

Histology and immunostaining for the androgen receptor $(A R)$. Mouse skeletons were fixed in $10 \%$ formalin for $24 \mathrm{~h}$ and then decalcified in $10 \%$ nitric acid overnight; other tissues were harvested and fixed in $10 \%$ formalin (Sigma, St. Louis, MO, USA), and all tissues were embedded in paraffin, sectioned, and stained with hematoxylin and eosin (H\&E). The H\&E-stained sections were observed by microscopy, and images were obtained using a SPOT Insight color camera (Nikon 80i, Tokyo, Japan).

AR (BioGenex F 39.4.1) immunostaining was performed using the two-step EnVision immunohistochemical procedure
(Dako, Glostrup, Denmark) as previously described (17). A monoclonal antibody against AR, which is present in prostate cancer, was used. Signals were visualized with DAB, and the slides were counterstained with Mayer's hematoxylin. AR-positive tissues were used as positive controls. PBS without the primary antibody was used as the negative control. Tumors were considered positive when $\geq 10 \%$ of the neoplastic cells were stained. The results of all specimens were scored by two independent observers.

Statistical analysis. Statistical differences and variances were evaluated with the t-test and variance analysis using statistical SPSS (SPSS, Inc., Chicago, IL, USA) software. Differences were considered significant at $\mathrm{P}<0.05$.

\section{Results}

Transfection efficiency of the LNCaP cells. After parental $\mathrm{LNCaP}$ cells were transfected with the lentiviral vector (PGK-luciferase-GFP), we visualized the expressed GFP at $24 \mathrm{~h}$ (the fluorescence intensity increased gradually with increasing passages). Transfection efficiency was determined by flow cytometry. Fig. 1A and B shows the proportion of cells expressing GFP and the fluorescence intensity. LNCaP-luc cells were polygonal in shape, and the fluorescence intensity was unequal. Flow cytometry detected GFP expression (Fig. 1C), and the transfection efficiency approached $80 \%$.

\section{Characteristics of the LNCaP cell subline}

Incidence of tumor formation. Table I summarizes the incidence of tumor formation in the nude mice inoculated s.c. with $2 \times 10^{6}$ LNCaP-luc cells and those inoculated with $0.5 \times 10^{6}$ LNCaP1-luc cells into the left ventricle of the heart. The development of primary tumors ( 8 of 20 for the LNCaP-luc cells, 16 of 20 for the LNCaP1-luc cells, respectively) by s.c injection and ( 2 of 20 for the LNCaP-luc cells, 9 of 20 for the LNCaP1-luc cells, respectively) by intracardiac injection was observed. When the LNCaP cell line was injected s.c. into nude mice, it induced tumors only at the site of injection; no metastases were detected. The pattern of the incidence of tumor formation differed between mice given s.c. injections 
Table I. Tumorigenicity and metastatic behavior of the LNCaP-luc and LNCaP1-luc cells injected either subcutaneously (s.c.) or into the left cardiac ventricle of nude mice.

\begin{tabular}{lcccc}
\hline Cell line & Mouse strain & Injection & Tumor formation (\%) & End-point (days) \\
\hline LNCaP-luc & Nude & s.c. & $8 / 20(40)$ & 45 \\
& & Intracardiac & $2 / 20(10)$ & 90 \\
LNCaP1-luc & Nude & s.c. & $16 / 20(80)$ & 45 \\
& & Intracardiac & $9 / 20(45)$ & 90 \\
\hline
\end{tabular}

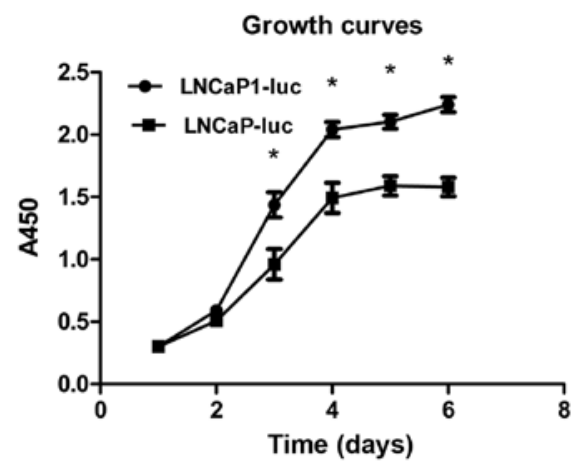

Figure 2. Cell growth curve of the LNCaP cell line as detected by CCK-8 assay. Experiments were repeated at least three times. Each data point represents the average value of triplicate wells and the standard deviation. The proliferative ability of LNCaP1-luc cells was markedly increased. There were statistical differences from day 3 to day $6\left({ }^{*} \mathrm{P}<0.05\right)$. The $\mathrm{x}$-axis indicates time (days) and the $y$-axis indicates optical density values at $450 \mathrm{~nm}$.

and those that received intracardiac injection of LNCaP-luc or LNCaP1-luc cells. Approximately 2-fold more mice injected with LNCaP1-luc cells developed tumors compared with mice injected with LNCaP-luc cells. Additionally, the tumor formation rate following intracardiac injection of LNCaP1-luc cells was higher than that after injection with LNCaP-luc cells. Thus, LNCaP1-luc cells injected into the heart had significantly higher tumorigenicity than did LNCaP-luc cells injected s.c.

Enhanced cell viability and growth of LNCaP1-luc cells compared to LNCaP-luc cells. Cell viability and growth kinetics were measured by the CCK- 8 assay. Growth curves of the two LNCaP cell line populations were generated to compare the growth characteristics after passage at various times. LNCaP1-luc cells grew more rapidly than did the LNCaP-luc cells (Fig. 2), indicating that LNCaP1-luc cells proliferated more rapidly. Additionally, the growth ability of the LNCaP1-luc cells increased gradually with the number of the passage (data not shown).

Enhanced tumorigenicity of LNCaPI-luc cells compared to the LNCaP-luc cell line. The prostate tumor growth rates of the two luciferase-expressing derivatives are shown in Fig. 3. External caliper measurements indicated that LNCaP1-luc cells grew at a faster rate than did the LNCaP-luc cells after s.c injection. After 5 weeks of growth in nude mice, the LNCaP1-luc tumors had reached a volume more than twice that of tumors derived from the LNCaP-luc cell line, suggesting that the LNCaP1-luc cells had a higher tumorigenicity.

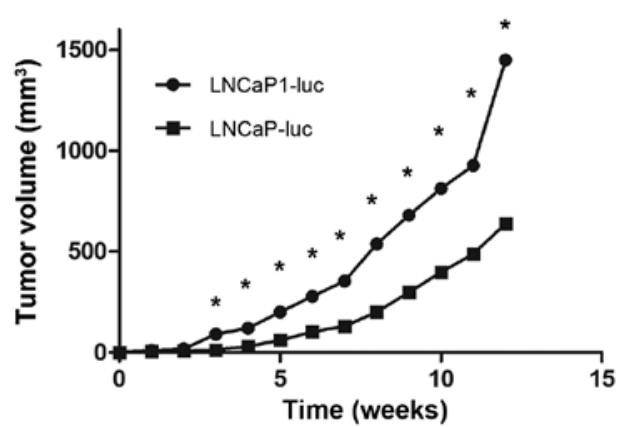

Figure 3. Prostate tumor growth is enhanced in mice injected s.c. with LNCaP1-luc cells. Cells were injected subcutaneously into nude mice, and mean tumor volumes over time were determined by caliper measurements. The LNCaP1-luc cell tumors grew more rapidly than did the LNCaP-luc cell tumors. There were statistical differences from day 3 to day $6\left({ }^{*} \mathrm{P}<0.05\right)$. The $\mathrm{x}$-axis indicates time (weeks) and the $\mathrm{y}$-axis indicates tumor volume $\left(\mathrm{mm}^{3}\right)$.

Increased invasiveness and induced epithelial-mesenchymal transition (EMT) in the LNCaP1-luc cell line. We found that the LNCaP1-luc cell line exhibited increased invasiveness when compared to the LNCaP-luc cell line; the relative percentage of LNCaP1-luc cells that migrated through the filters compared to LNCaP-luc cells was $276.2 \pm 26.4 \%$ as observed by Transwell migration assay (Fig. 4A). mRNA and protein expression of the epithelial cell marker E-cadherin was significantly lower, whereas mRNA and protein expression of the mesenchymal cell marker vimentin was higher in the LNCaP1-luc cells when compared to the LNCaP-luc cells (Fig. 4B and C). This may explain why the LNCaP1-luc cell line exhibited increased invasiveness when compared to the LNCaP-luc cell line. The Snail mRNA level was higher in the LNCaP1-luc cell line, which is crucial for the EMT process, whereas Slug mRNA expression did not differ between the LNCaP1-luc and the LNCaP-luc cells.

Bioluminescence imaging after intracardiac injection. We injected LNCaP-luc or LNCaP1-luc cells into the left cardiac ventricle of male nude mice at a rate of $0.5 \times 10^{6}$ cells/mouse. Since the LNCaP cell line was labeled with luciferase and GFP, we were able to observe metastatic tumors by bioluminescence imaging. The mice were imaged weekly beginning 4 weeks after the intracardiac injection. The whole mouse image in Fig. 5 shows the results of the injection of LNCaP-luc and LNCaP1-luc cells. A small hot spot of bioluminescence, which increased with time, was observed following LNCaP-luc cell injection (Fig. 5A). Fig. 5B shows the localized and distant metastasized cancer in the LNCaP1-luc heart-injected animal 

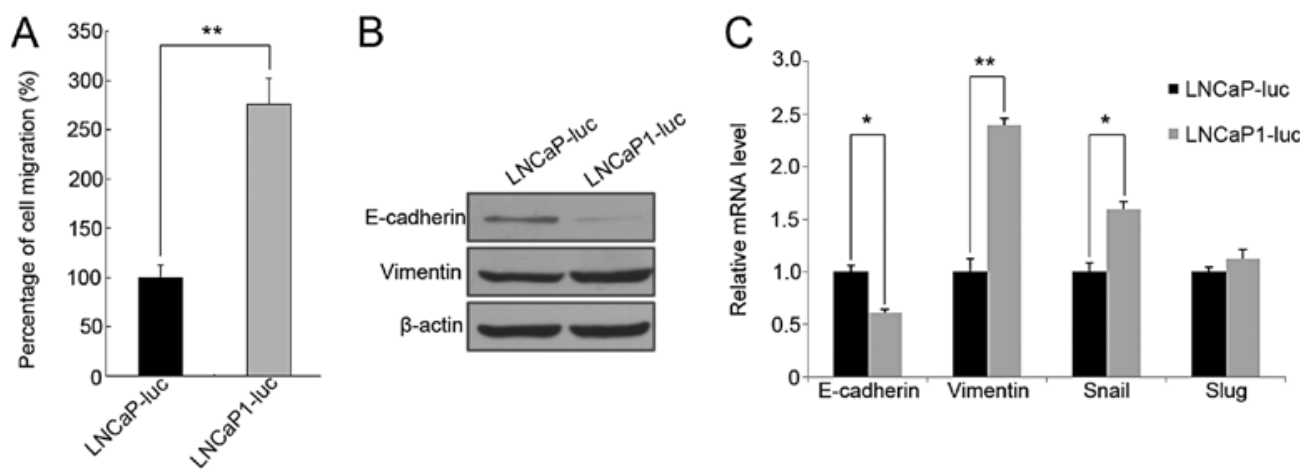

Figure 4. Results of the Transwell assay, western blot analysis and RT-PCR analysis. (A) Transwell assay shows that the relative percentage of LNCaP1-luc cells that migrated through the filters was $276.2 \pm 26.4 \%$. (B) Western blot analysis shows that the changes in the protein expression profile between LNCaP-luc and LNCaP1-luc cells were closely associated with epithelial to mesenchymal transition. (C) RT-PCR of the mRNA expression of E-cadherin, vimentin, Snail and Slug in the LNCaP-luc and LNCaP1-luc cells; ${ }^{* *} \mathrm{P}<0.001,{ }^{*} \mathrm{P}<0.05$.

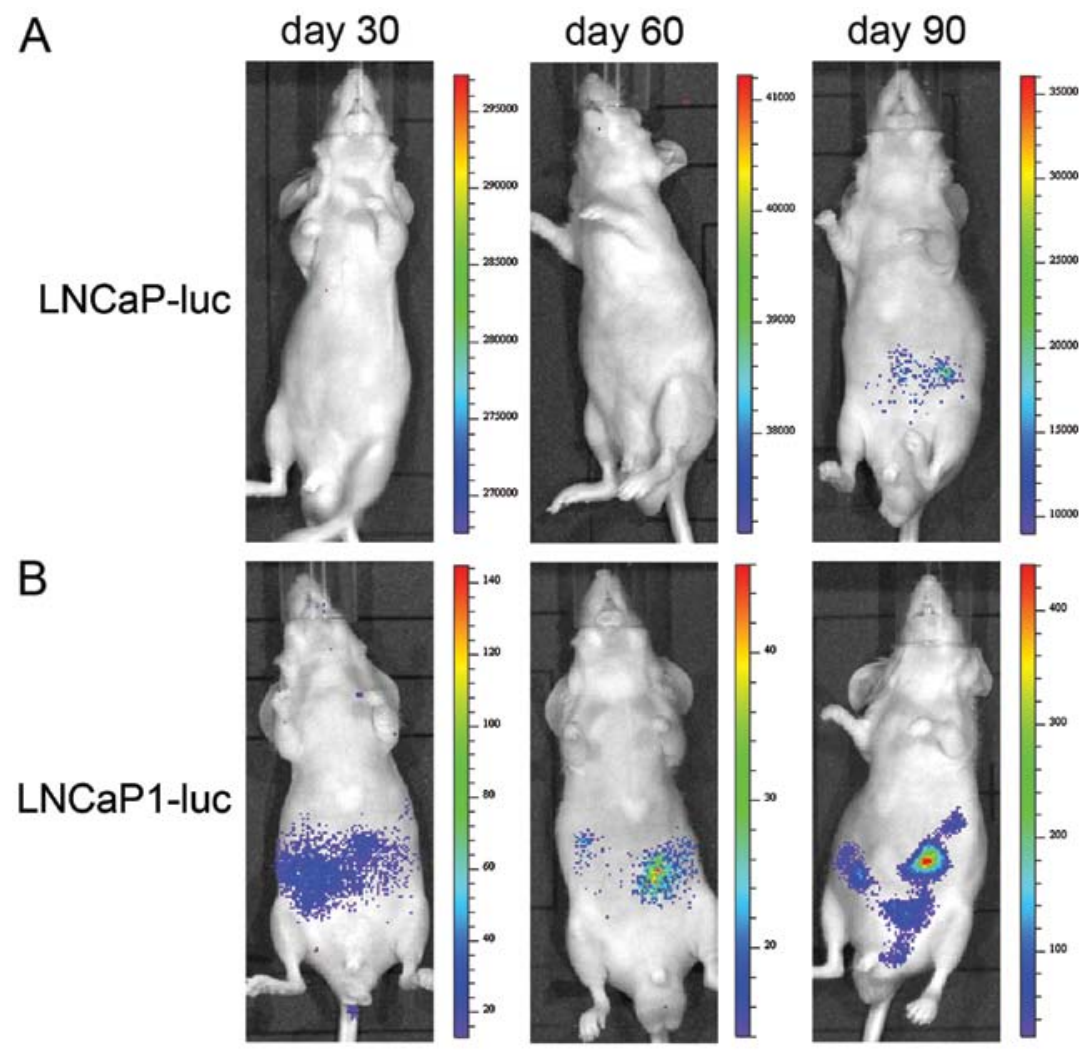

Figure 5. Tumor growth and multiple metastases were monitored over time by in vivo bioluminescence imaging. Data for a representative mouse are shown. (A) No visible metastases were initially observed in vivo following inoculation with the LNCaP-luc cell line. A small hot-spot of bioluminescence appeared over time. (B) Induction of multiple localized and distant metastases in vivo after injection of LNCaP1-luc cells into the heart of nude mice. Colored bar indicates the bioluminescence signal intensity range (photon $/ \mathrm{sec} / \mathrm{cm}^{2} /$ steradian).

model; transformation of a hot-spot of the bioluminescence signal in the lower abdomen was widely distributed over time. Thus, the LNCaP1-luc cell line induced multiple metastases after intracardiac inoculation. In contrast, the inoculation of LNCaP-luc cells rarely induced such metastases. This result suggests that LNCaP1-luc cells have a higher metastatic potential than their parental LNCaP-luc cells.

Histology and immunohistochemistry of mouse tissues. Mouse tissues were also evaluated by histological and immunohistochemical analyses. Mice were decalcified and processed for conventional histological and immunohistochemistry examination 90 days after injection. Representative histological sections of the lung (A), liver (B), skeletal (C), kidney (D), heart tissues $(\mathrm{E})$, and lymph nodes $(\mathrm{F})$ and metastases are shown in Fig. 6. Tumor cells had a nucleus larger (stained blue) than that in the normal cells. The lung lobes, kidneys, and hepatic lobules were full of tumor cells. Additionally, slight tumor growth was observed along the needle tract in the heart (arrow). The groin and para-vertebral lymph nodes were nearly infiltrated by tumor cells, and the normal structure of the lymph nodes could not be seen. The prostatic origin of the 

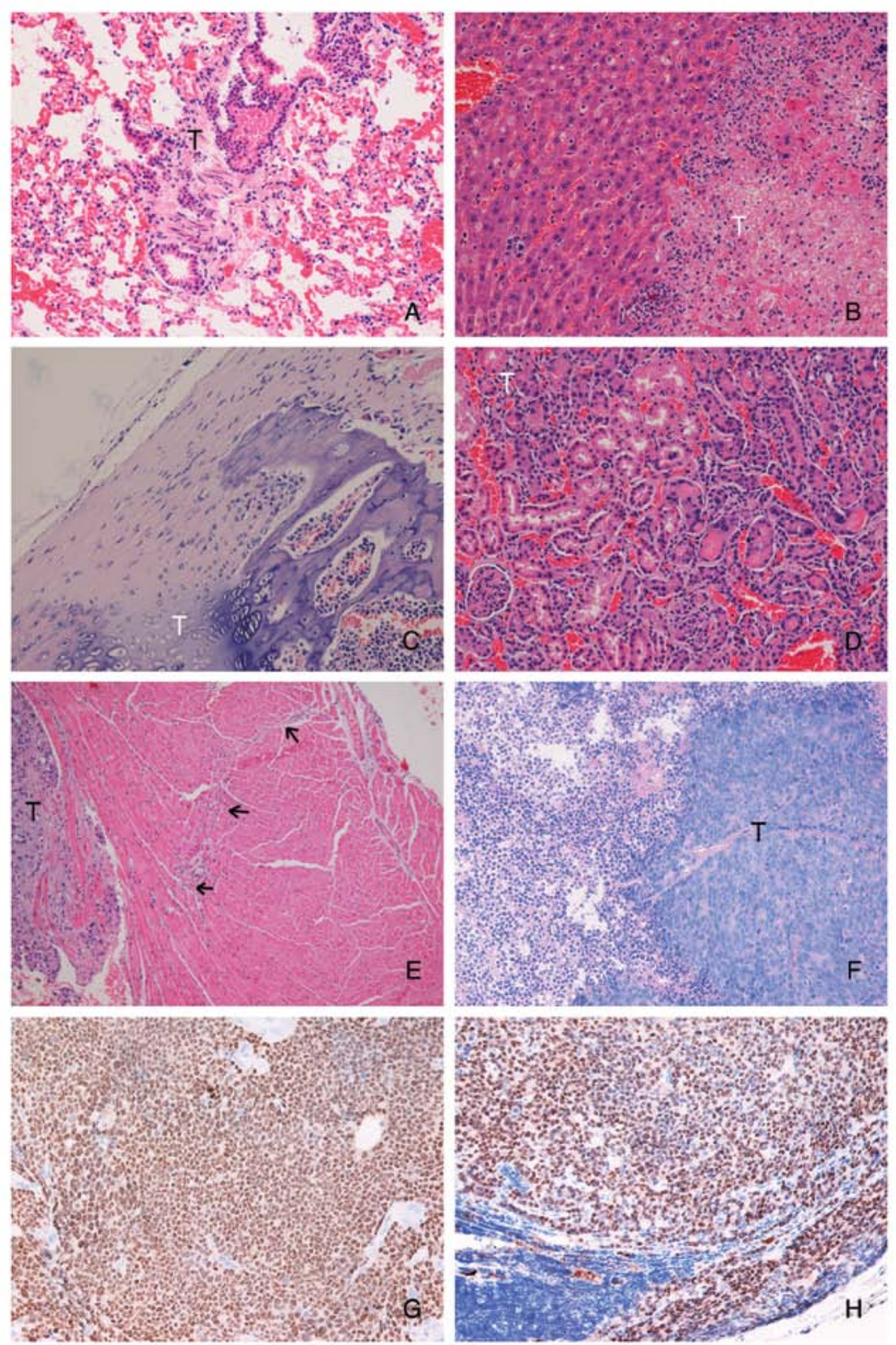

Figure 6. Hematoxylin and eosin and immunohistochemical staining of mouse tissue sections at the end of the study period. Representative histological sections of (A) lung (B) liver (C) skeletal (D) kidney (E) heart tissues and (F) lymph nodes showing metastases. (G and H) Immunohistochemical staining for the androgen receptor (AR) shows subcutaneous and lymph node tumors. $(\mathrm{H})$ Positive staining is noted in the tumor cell nuclei, but not in infiltrating lymphocytes in and around the tumor nests. T, tumor cells. Arrow shows tumor growth along the needle tract.

epithelial cells derived from the s.c. tumors in the lymph nodes of male nude mice was confirmed to be positive for AR, but the staining was scattered (Fig. 6G and H); all other metastatic sublines showed similar immunostaining (data not shown). The results of the immunohistochemical analysis suggest that all sublines were human and shared a common AR marker with the parental LNCaP cells.

\section{Discussion}

Experimental animal models of human cancers are required to elucidate the molecular events associated with prostatic carcinogenesis and progression. In vitro testing provides a significant amount of information on cellular characterization but is inadequate to provide a full understanding of bone meta- static processes. As we know, at present few similar animal models are available for studying the progression of human prostate cancer from the primary tumor to metastasis. We described the establishment of such an animal model in this study. We generated LNCaP1-luc cells which showed multiple metastases in mice when injected into the left cardiac muscle. Notably, the characteristics of LNCaP1-luc cells differed from those of the parental cells, and showed increased cell proliferation, cell invasiveness, tumorigenicity, and metastastic potential and underwent EMT.

Human prostate cancer is initially androgen dependent, but it acquires the ability to grow in the absence of androgens after androgen-ablation therapy. The AR is expressed in the majority of primary and metastatic prostate cancers (19). $\mathrm{LNCaP}$ cells are an androgen-responsive human prostate 
cancer cell line derived originally from a lymph node with prostate cancer metastasis (20). LNCaP cells express the AR, and their growth is stimulated by androgens (21). Many studies have suggested that the AR gene is amplified in bone metastases from an androgen-dependent state to hormone-refractory prostate cancer and that it requires active AR signaling during prostate cancer progression (22). Not many models have been described for the LNCaP human prostate cancer cell line in nude mice. We injected LNCaP1-luc cells directly into the left heart ventricle of nude mice, which resulted in successful development of a multiple metastatic animal model. Moreover, the AR was detected in LNCaP cells (Fig. 6G and H) and may provide a foundation for further study of AR-targeted anticancer therapy drugs (23-26).

The LNCaP1-luc cell subline metastasized to the lymph nodes, bones, lungs and liver with an incidence of 10-45\% (Table I). This tumor formation rate is higher than that previously reported $(2,27)$. There are several possible explanations for, and potential implications of, these findings. First, the characteristics of the LNCaP sublines differed from those of the primary line. LNCaP1-luc cells were obtained using the LNCaP-luc prostate carcinoma cell line following repeated subcutaneous injections with Matrigel in nude mice, which increased cell tumorigenicity as mentioned in numerous studies $(28,29)$, followed by isolation of the cells from tumors. Subcutaneous tumor volume measured using external calipers indicated that LNCaP1-luc cells grew more rapidly than did LNCaP-luc cells (Fig. 2) (30). The CCK-8 assay results also showed that the growth ability of LNCaP1-luc cells was enhanced when compared with LNCaP-luc cells (Fig. 3). Thus, the LNCaP1-luc cells exhibited a higher tumor formation rate than did LNCaP-luc cells. Second, E-cadherin expression was used to monitor the epithelial phenotype, and its loss suggests EMT (16). The EMT triggers tumor metastasis and enhances metastatic potential $(31,32)$. The results of the western blot analysis and PCR suggested that LNCaP1-luc cells may have induced LNCaP-luc cells to undergo EMT through the Snail transcription factor. This result is consistent with many other studies $(33,34)$. At the same time, the Transwell assay results illustrated that $\mathrm{LNCaP1-luc}$ cells were more invasive than LNCaP-luc cells. Therefore, LNCaP1-luc cells may have greater potential for bone metastasis. Indeed, our results support a number of reports of the importance of EMT in cancer metastases and the mechanisms involved (33,35-39). These studies demonstrated that prostate cells undergoing EMT become more invasive and express several genes associated with metastasis. Third, the technique most widely used for detection of metastasis is radiological examination. However, it should be noted that micrometastases elude radiographic detection, and cancer tends to be diagnosed at later stages, reducing access to appropriate therapeutic facilities and drugs and adversely affecting survival. In this study, we used bioluminescence imaging to visualize cancer growth, tumor cell motility, and invasion. Wetterwald et al (14) reported that whole-body bioluminescence reporter imaging detects microscopic bone marrow metastases with volumes of $\sim 0.5 \mathrm{~mm}^{3}$ when used with luciferase-transfected cells. This sensitive technology for early detection of tumor growth is preceded by the appearance of a radiologically evident metastasis in $\sim 2$ weeks. Thus, applying bioluminescence imaging allows detection of micrometastases at the very early stage, which improves the detection rate. In summary, the LNCaP1-luc cell line exhibited high metastatic potential which facilitated the generation of a metastatic animal model of prostate cancer. We obtained the following evidence for metastasis: i) monitoring of tumor growth and multiple metastases over time in vivo by bioluminescence imaging; and ii) histological and immunohistochemical data.

In short, our results have various differences compared with other previously reported results. The use of this method yielded a cell line with greater invasive potential. Thus, using these LNCaP1-luc cells can generate an animal model of multiple metastatic prostate cancers.

\section{Acknowledgements}

This work was supported by Science and Technology Planning Project of Guangdong Province (No. 2010B060500005), Guangdong Key Natural Science Foundation (No. 8251008901000009), and National Natural Science Foundation of China (No. 30970857).

\section{References}

1. Marech I, Vacca A, Ranieri G, Gnoni A and Dammacco F: Novel strategies in the treatment of castration-resistant prostate cancer (Review). Int J Oncol 40: 1313-1320, 2012.

2. Jemal A, Bray F, Center MM, Ferlay J, Ward E and Forman D: Global cancer statistics. CA Cancer J Clin 61: 69-90, 2011.

3. Wang Q, Li W, Zhang Y, et al: Androgen receptor regulates a distinct transcription program in androgen-independent prostate cancer. Cell 138: 245-256, 2009.

4. Power CA, Pwint H, Chan J, et al: A novel model of bonemetastatic prostate cancer in immunocompetent mice. Prostate 69: 1613-1623, 2009.

5. Zuluaga MF, Gabriel D and Lange N: Enhanced prostate cancer targeting by modified protease sensitive photosensitizer prodrugs. Mol Pharm 9: 1570-1579, 2012.

6. Pienta KJ, Abate-Shen C, Agus DB, et al: The current state of preclinical prostate cancer animal models. Prostate 68: 629-639, 2008.

7. Rosol TJ, Tannehill-Gregg SH, LeRoy BE, Mandl S and Contag CH: Animal models of bone metastasis. Cancer 97: 748-757, 2003.

8. Blouin S, Basle MF and Chappard D: Rat models of bone metastases. Clin Exp Metastasis 22: 605-614, 2005.

9. Singh AS, Macpherson GR, Price DK, Schimel D and Figg WD: Evaluation of human fetal bone implants in SCID mice as a model of prostate cancer bone metastasis. Oncol Rep 15: 519-524, 2006

10. Song H, Shahverdi K, Huso DL, et al: An immunotolerant HER-2/neu transgenic mouse model of metastatic breast cancer. Clin Cancer Res 14: 6116-6124, 2008.

11. Arguello F, Baggs RB and Frantz CN: A murine model of experimental metastasis to bone and bone marrow. Cancer Res 48: 6876-6881, 1988.

12. Jenkins DE, Hornig YS, Oei Y, Dusich J and Purchio T: Bioluminescent human breast cancer cell lines that permit rapid and sensitive in vivo detection of mammary tumors and multiple metastases in immune- deficient mice. Breast Cancer Res 7: R444-R454, 2005.

13. Fritz V, Louis-Plence P, Apparailly F, et al: Micro-CT combined with bioluminescence imaging: a dynamic approach to detect early tumor-bone interaction in a tumor osteolysis murine model. Bone 40: 1032-1040, 2007.

14. Wetterwald A, van der Pluijm G, Que I, et al: Optical imaging of cancer metastasis to bone marrow: a mouse model of minimal residual disease. Am J Pathol 160: 1143-1153, 2002.

15. Xing N, Chen Y, Mitchell SH and Young CY: Quercetin inhibits the expression and function of the androgen receptor in $\mathrm{LNCaP}$ prostate cancer cells. Carcinogenesis 22: 409-414, 2001. 
16. Emadi BM, Soheili ZS, Schmitz I, Sameie S and Schulz WA: Snail regulates cell survival and inhibits cellular senescence in human metastatic prostate cancer cell lines. Cell Biol Toxicol 26: 553-567, 2010

17. Chen JN, Jiang Y, Li HG, et al: Epstein-Barr virus genome polymorphisms of Epstein-Barr virus-associated gastric carcinoma in gastric remnant carcinoma in Guangzhou, Southern China, an endemic area of nasopharyngeal carcinoma. Virus Res 160 191-199, 2011.

18. Yang M, Jiang P, Sun FX, et al: A fluorescent orthotopic bone metastasis model of human prostate cancer. Cancer Res 59: 781-786, 1999.

19. Guo Z, Yang X, Sun F, et al: A novel androgen receptor splice variant is up-regulated during prostate cancer progression and promotes androgen depletion-resistant growth. Cancer Res 69: 2305-2313, 2009.

20. Wu HC, Hsieh JT, Gleave ME, Brown NM, Pathak S and Chung LW: Derivation of androgen-independent human LNCaP prostatic cancer cell sublines: role of bone stromal cells. Int $\mathbf{J}$ Cancer 57: 406-412, 1994.

21. Hobisch A, Culig Z, Radmayr C, Bartsch G, Klocker H and Hittmair A: Distant metastases from prostatic carcinoma express androgen receptor protein. Cancer Res 55: 3068-3072, 1995.

22. Brown RS, Edwards J, Dogan A, et al: Amplification of the androgen receptor gene in bone metastases from hormonerefractory prostate cancer. J Pathol 198: 237-244, 2002.

23. Ullen A, Farnebo M, Thyrell L, et al: Sorafenib induces apoptosis and autophagy in prostate cancer cells in vitro. Int J Oncol 37: $15-20,2010$.

24. Liu Y, Wu X, Dong Z and Lu S: The molecular mechanism of Vav3 oncogene on upregulation of androgen receptor activity in prostate cancer cells. Int J Oncol 36: 623-633, 2010

25. Martin TA and Jiang WG: Anti-cancer agents in medicinal chemistry (Formerly current medicinal chemistry - Anti-cancer agents). Anticancer Agents Med Chem 10: 1, 2010.

26. Sturge J, Caley MP and Waxman J: Bone metastasis in prostate cancer: emerging therapeutic strategies. Nat Rev Clin Oncol 8: 357-368, 2011 .

27. Wu TT, Sikes RA, Cui Q, et al: Establishing human prostate cancer cell xenografts in bone: induction of osteoblastic reaction by prostate-specific antigen-producing tumors in athymic and $\mathrm{SCID} / \mathrm{bg}$ mice using LNCaP and lineage-derived metastatic sublines. Int J Cancer 77: 887-894, 1998.
28. Kleinman HK and Martin GR: Matrigel: basement membrane matrix with biological activity. Semin Cancer Biol 15: 378-386, 2005.

29. Tilghman RW, Blais EM, Cowan CR, et al: Matrix rigidity regulates cancer cell growth by modulating cellular metabolism and protein synthesis. PLoS One 7: e37231, 2012.

30. Thalmann GN, Anezinis PE, Chang SM, et al: Androgenindependent cancer progression and bone metastasis in the LNCaP model of human prostate cancer. Cancer Res 54: 2577-2581, 1994.

31. Xu J, Wang R, Xie ZH, et al: Prostate cancer metastasis: role of the host microenvironment in promoting epithelial to mesenchymal transition and increased bone and adrenal gland metastasis. Prostate 66: 1664-1673, 2006.

32. Yang J and Weinberg RA: Epithelial-mesenchymal transition: at the crossroads of development and tumor metastasis. Dev Cell 14: 818-829, 2008.

33. Kogan-Sakin I, Tabach Y, Buganim Y, et al: Mutant p53(R175H) upregulates Twist1 expression and promotes epithelial-mesenchymal transition in immortalized prostate cells. Cell Death Differ 18: 271-281, 2011.

34. Kong D, Banerjee S, Ahmad A, et al: Epithelial to mesenchymal transition is mechanistically linked with stem cell signatures in prostate cancer cells. PLoS One 5: e12445, 2010.

35. Birchmeier C, Birchmeier W and Brand-Saberi B: Epithelialmesenchymal transitions in cancer progression. Acta Anat 156: 217-226, 1996.

36. Grille SJ, Bellacosa A, Upson J, et al: The protein kinase Akt induces epithelial mesenchymal transition and promotes enhanced motility and invasiveness of squamous cell carcinoma lines. Cancer Res 63: 2172-2178, 2003.

37. Guarino M, Rubino B and Ballabio G: The role of epithelialmesenchymal transition in cancer pathology. Pathology 39: 305-318, 2007.

38. Larue L and Bellacosa A: Epithelial-mesenchymal transition in development and cancer: role of phosphatidylinositol 3 kinase/AKT pathways. Oncogene 24: 7443-7454, 2005.

39. Wells A, Yates C and Shepard CR: E-cadherin as an indicator of mesenchymal to epithelial reverting transitions during the metastatic seeding of disseminated carcinomas. Clin Exp Metastasis 25: 621-628, 2008 